\title{
Pulmonary infiltrates in adult acute leukaemia: empirical treatment or lung biopsy?
}

\author{
AG WARDMAN, NJ COOKE
}

The prognosis of adult acute leukaemia has improved with better chemotherapy and the use of newer antibiotics for the infective problems of neutropenia. Pulmonary complications are second in importance only to disseminated infection as the major cause of morbidity and mortality, though they are themselves frequently accompanied by septicaemia. ${ }^{2}$ The range of pulmonary disease includes Gram negative and Gram positive pneumonias, opportunistic infections particularly with fungi and Pneumocystis carinii, leukaemic infiltration, pulmonary haemorrhage and oedema; two or more of these processes may be present..$^{34}$ Opinion is divided on whether the lung or the gastrointestinal tract is the more frequent source of septicaemia in neutropenic patients. ${ }^{256}$ The reported mortality rate associated with pulmonary complications is around $60-65 \% .^{78}$ Death may occur within a few days of the appearance of an abnormality on a chest radiograph. ${ }^{9}$

In vivo and postmortem studies agree that Gram negative organisms are the commonest cause of pneumonia, though Gram positive bacteria and anaerobes are also important. ${ }^{1-3} 10$ The incidence of any one organism may vary from hospital to hospital, depending on the particular bacterial population prevalent at the time. The incidence of fungal pneumonia has been found to be as high as $25 \%{ }^{8}$ in a series which included postmortem data, but a figure of around $12 \%{ }^{10}$ may be more representative. Although $19 \%$ of the infiltrates in a recent series from the United States ${ }^{11}$ were due to Aspergillus or Candida, this was not an unselected consecutive series. Pneumocystis carinii pneumonia occurs less often in adults than in children with acute leukaemia, but it is a well documented occurrence. ${ }^{1213}$ There is a clinical impression that opportunistic infections are less common in British practice, but there is no large in vivo or necropsy series to confirm this. Indeed, it was the continuing high mortality associated with pulmonary infiltration in acute leukaemia that directed investigators in the

Address for reprint requests: Dr NJ Cooke, Department of Respiratory Medicine, General Infirmary, Leeds LS1 3EX.
United States to seek and identify the causes, particularly the opportunistic infections, which are difficult to diagnose by conventional techniques.

Microscopic leukaemic infiltration in postmortem studies is common- $64 \%$ in one series ${ }^{4}$ - but in only a few patients does it occur on a macroscopic and radiologically detectable scale. Such infiltration tends to develop in patients with a poorly responding leukaemia, whose prognosis is limited by the underlying disease. Pulmonary haemorrhage is usually associated with a low platelet count ${ }^{4}$ and may be extensive. ${ }^{14}$ It is difficult to diagnose in vivo, though techniques avoiding biopsy have been reported. ${ }^{15}$ Correction of the bleeding tendency with platelet transfusion should theoretically stop intrapulmonary haemorrhage, and this complication must be considered a potentially remediable condition.

With this knowledge, how should a clinician managing an adult with acute leukaemia respond to the finding of an abnormal chest radiograph? Should he pursue an invasive investigatory approach in an attempt to make a specific diagnosis in all cases? Would he be better just to treat empirically with antibiotics including high dose co-trimoxazole and antifungal agents? Certainly conventional investigations, including sputum examination and blood cultures, give positive results in less than half of the cases $^{8} 10$ and the results may not be available for 24-48 hours. During this time empirical treatment may have to be prescribed in view of the speed with which death can occur. Whereas a platelet count of less than $10 \times 10^{9} / 1$ might indicate the need for platelet transfusion for possible intrapulmonary haemorrhage, this is only rarely the main cause of pulmonary infiltration.

Few hospitals, even large ones, can have the facilities available at only a few hours' notice every day of the year to perform fibreoptic bronchoscopy and its associated procedures, open lung biopsy, or transtracheal aspiration and analyse the brushings, washings, and biopsy specimens that are necessary; the financial implications of such a service must be considered before routine use is justified. Enthusiasts for an aggressive invasive policy must consider whether the advantages of making a 
specific bacteriologically or pathologically proved diagnosis justify the penalties. They must take account of the complications of the procedures (which may have to be tepeated if further infiltrates appear at a later date) and be able to show a lower mortality rate in the episodes than with empirical treatment started after all the clinical laboratory and radiological evidence has been taken into account. Is there any evidence that invasive procedures have influenced mortality? Certainly they have helped identify the causes of pulmonary infiltration in vivo and this may in itself have influenced the empirical approach to management.

One of the difficulties in attempting to answer these questions lies paradoxically in the number and variety of the reports available for analysis. Unfortunately in only a few instances has a particular invasive procedure been assessed in adult acute leukaemia - many series are comprised of a mix of immunosuppressed patients, including those with lymphomas, renal and marrow transplants, and solid tumours. For example, an analysis of 14 series (not exhaustive but representative) from 1973 to $1981,7^{81116^{-26}}$ of which all but two ${ }^{25}$ date from 1977 and of which only two are British, ${ }^{22}{ }^{26}$ reveals that $359(48 \%)$ of the 753 patients had leukaemia, of whom only $268(36 \%)$ had acute leukaemia. Three reports ${ }^{81126}$ contain $184(67 \%)$ of the total cases of acute leukaemias. In addition, probably 13 of the 14 series contained selected patients and eight were retrospective. The time interval between presentation with a radiological abnormality and the invasive procedure was up to nine days ${ }^{23}$ and it is thus likely that some patients died during this period. In some series the procedures were carried out only after conventional investigations had shown negative results or the investigators only analysed information on those patients referred to a surgical service for lung biopsy. Hence the reported relative frequency of a particular infection or infiltration in many of the studies is of no absolute relevance, and in addition the use of radiation and of bleomycin and other drugs must have widened the diagnostic possibilities.

These series have, however, led to broad conclusions on the complication rates and specific diagnostic rates of the various biopsy procedures, including cutting needle, transbronchial and open biopsy. Cutting needle biopsy has unacceptable complication rates of pneumothorax and bleeding ${ }^{11} 1627$ and has been abandoned in most centres. Open biopsy has a higher specific diagnostic rate of 65-70\%, compared with around $50 \%$ for transbronchial biopsy. Even though open biopsy can be performed through a 4-8 cm incision, the postoperative care required and the discomfort to the patient are considerably grea- ter than with the transbronchial route. Whether open biopsy should be reserved for those patients in whom a specific diagnosis has not been made by transbronchial biopsy or should be used as the definitive initial diagnostic procedure is debatable, and there is evidence to support both viewpoints. ${ }^{21}{ }^{23}$ Even with open lung biopsy no specific pathological diagnosis will be made in up to $30 \%$ of patients.

Arguably the least invasive of the invasive procedures is transtracheal aspiration. ${ }^{28}$ Application of this technique in a recent British study on 44 patients with acute leukaemia and four with myeloma $^{26}$ with profound neutropenia, fever, and respiratory symptoms resulted in a positive bacterial isolate in $56 \%$. Although two cases of aspergillus infection were identified, it is not claimed that this technique is designed to identify opportunistic infection. A great advantage of the method is the ability to isolate pathogenic bacteria in patients with no sputum or even no radiological abnormality. All patients in whom a positive culture was obtained before the start of empirical antibiotic treatment responded to an appropriate antibiotic; two thirds, however, died when a culture was positive in patients already on antibiotic treatment. This could be related to the differing rates of isolation of coliforms as the commonest bacteria. Coliforms were found in 10 of 16 patients already having antibiotics compared with one of 11 patients not having antibiotics. Was the empirical use of antibiotics the reason for this or were the coliforms the primary bacterial infection, the patient being investigated only because of a failure to respond to the initial antibiotic regime? If the findings of this study are confirmed and if further bacterial investigations support the contention that the coliforms were secondary colonisers, then the empirical antibiotic approach for respiratory infection in these circumstances must be reassessed.

Though there may be some benefit to the patient from early transtracheal aspiration, it is difficult to find evidence of benefit from the more invasive procedures in terms of a lower mortality rate, even where a specific diagnosis was made and treatment altered. ${ }^{17} 182930$ One recent study of the usefulness of transbronchial biopsy in a mixed group of immunosuppressed patients showed that, even though $65 \%$ had a change of therapeutic approach as a result of the biopsy, making a specific diagnosis did not improve survival. ${ }^{29}$ The matter is unlikely to become any clearer until a major prospective study in adult leukaemia is undertaken that compares an unselected consecutive series managed either (1) by empirical treatment, possibly preceded by transtracheal aspiration, with the option of a later invasive procedure if progress is not satisfactory, or (2) 
by an early initial biopsy procedure followed by the appropriate treatment indicated. Any such study would encounter the problem of how to manage the seriously ill, hypoxaemic and thrombocytopenic patient who may present during the night or weekend; it would be difficult to withhold antibiotic treatment while arrangements were made for an invasive biopsy procedure.

An attempt to rationalise the management of this clinical condition came from the findings of a retrospective review analysing the aetiology of episodes of pulmonary infiltration and the extent of radiologically apparent disease in 139 adults with leukaemia, of whom 85 had acute leukaemia." In those with local radiological abnormalities $74 \%$ of the infiltrations were infective, $87 \%$ of the infections being bacterial; opportunistic infections occurred in only $13 \%$ of episodes. In diffuse radiological disease $65 \%$ of episodes were not infective, though $93 \%$ of the infective episodes were opportunistic. On this evidence biopsy procedures to diagnose opportunistic infections would seem most appropriate in patients with diffuse changes in whom there was reason to suspect a predominantly infectious aetiology rather than pulmonary haemorrhage, leukaemic infiltration, or pulmonary oedema. The main criticisms of this important contribution are the loose definition of the two radiological groups and the influence of delay in referral from another hospital that may have resulted in selection of patients-particularly in the "diffuse" group since the mortality in patients with diffuse changes affecting all lung zones is high in the first 48 hours after the first detection of an abnormal chest radiograph. ${ }^{9}$

A scheme of initial management to be considered might be: (1) The routine use of transtracheal aspiration as soon as possible after the onset of fever and respiratory symptoms, whether there are radiological abnormalities or not. (2) The administration of broad spectrum antibiotic combinations until the culture results are available, transfusion of platelets if the count is less than $10 \times 10^{9} / 1$, and the addition of diuretic treatment if pulmonary oedema is suspected. (3) The use of transbronchial biopsy in patients with "local" radiological changes if progress is not satisfactory after 48 hours. (4) Empirical treatment, which should probably include high dose co-trimoxazole and amphotericin $B$, in patients with diffuse changes, a lung biopsy being performed in the first 24 hours if possible, depending on the clinical state of the patient. Physicians require some hard evidence from clinical trials that a lung biopsy is likely to affect outcome in these circumstances.

Until proper trials are designed to compare different patterns of management it is doubtful if further progress can be made in improving the prog- nosis of lung disease accompanying adult acute leukaemia.

\section{References}

' Tobias JS, Wrigley PFM, O' Grady F. Bacterial infection and acute myeloblasic leukaemia: an analysis of two hundred patients undergoing intensive remission induction therapy. Europ J Cancer 1978;14:383-91.

${ }^{2}$ Slevin M, Rohatiner A, Malpas JS, Lister A. Pneumonia during treatment of acute leukaemia. $\mathrm{Br}$ Med J 1981;282:473.

${ }^{3}$ Chang H-Y, Rodriguez V, Narboni G, Bodey GP, Luna MA, Freireich EJ. Causes of death in adults with acute leukaemia. Medicine (Baltimore) 1976;55:259-68.

${ }^{4}$ Bodey GP, Powell RD, Hersh EM, Yetevian A, Freireich EJ. Pulmonary complications of acute leukaemia. Cancer 1966;19:781-93.

5 The EORTC International Antimicrobial Therapy Project Group. Three antibiotic regimens in the treatment of infection in febrile granulocytopenic patients with cancer. J Infect Dis 1978;137:14-29.

- Hayhoe FGJ, Rees JKH. Pneumonia during treatment of acute leukaemia. $\mathrm{Br}$ Med J 1980;281:1566-7.

7 Singer C, Armstrong D, Rosen PP, Walzer DP, Yu B. Diffuse pulmonary infiltrates in immunosuppressed patients. Am J Med 1979;66:110-9.

${ }^{8}$ Sickles EA, Young VM, Greene WH, Wiernick PH. Pneumonia in acute leukaemia. Ann Intern Med 1973;79:528-34.

9 Wardman AG, Milligan DW, Child JA, Delamore IW, Cooke NJ. Pulmonary infiltrates and adult acute leukaemia-empirical treatment and survival related to the extent of pulmonary radiological disease. Thorax 1984;39:568-71.

${ }^{10}$ Bodey GP, Rodriguez V, Chang H-Y, Narboni G. Fever and infection in leukemic patients. Cancer 1978;41: 1610-22.

"Tenholder MF, Hooper RG. Pulmonary infiltrates in leukaemia. Chest 1980;78:468-73.

12 Rosen PP, Martini N, Armstrong D. Pneumocystis carinii pneumonia. Am J Med 1975;58:794-802.

${ }^{13}$ Michaelis LL, Leight GS, Powell RD, DeVita VT. Pneumocystis pneumonia: the importance of early open lung biopsy. Ann Surg 1976;183:301-6.

14 Blank N, Castellino RA, Shah V. Radiographic aspects of pulmonary infection in patients with altered immunity. Radiol Clin North Am 1973;11:175-90.

${ }^{15}$ Golde DW, Drew WL, Klein HZ, Finley TN, Cline MJ. Occult pulmonary haemorrhage in leukaemia. $\mathrm{Br} \mathrm{Med}$ J 1975; ii:166-8.

${ }^{16}$ Cunningham JH, Zavala DC, Corry RJ, Keim LW. Trephine air drill, bronchial brush, and fibreoptic transbronchial lung biopsies in immunosuppressed patients. Am Rev Respir Dis 1977;115:213-20.

17 Pennington JE, Feldman NT. Pulmonary infiltrates and fever in patients with haematologic malignancy: assessment of transbronchial biopsy. Am J Med 1977;62:581-7.

${ }^{18}$ Rossiter SJ, Craig Miller D, Chung AM, Carrington CB, Mark JBD. Open lung biopsy in the immunosuppressed patient-is it really beneficial? $J$ Thorac Cardiovasc Surg 1979;77:338-45.

19 Lauver GL, Hasan FM, Morgan RB, Campbell SC. The usefulness of fibreoptic bronchoscopy in evaluating 
new pulmonary lesions in the compromised host. Am J Med 1979;66:580-5.

${ }^{20}$ Feldman NT, Pennington JE, Ehrie MG. Transbronchial lung biopsy in the compromised host. JAMA 1977; 238: 1377-9.

${ }^{21}$ Toledo-Pereyra LH, DeMeester TR, Kinealey A, MacMahon H, Churg A, Golomb H. The benefits of open lung biopsy in patients with previous non-diagnostic transbronchial lung biopsy: a guide to appropriate therapy. Chest 1980;77:647-50.

${ }^{22}$ Phillips MJ, Knight RK, Green M. Fibreoptic bronchoscopy and diagnosis of pulmonary lesions in lymphoma and leukaemia. Thorax 1980;35:19-25.

${ }^{23}$ Leight GS, Michaelis SLL. Open lung biopsy for the diagnosis of acute diffuse pulmonary infiltrates in the immunosuppressed patient. Chest 1978;73:477-82.

${ }^{24}$ Matthay RA, Farmer WC, Odero D. Diagnostic fibreoptic bronchoscopy in the immunocompromised host with pulmonary infiltrates. Thorax 1977;32:539-45.
${ }^{25}$ Greenman RL, Goodall PT, King D. Lung biopsy in immunocompromised hosts. Am J Med 1975;59:488-96.

${ }^{26}$ Slevin ML, Bell LR, Catto-Smith AG, Ford JM, Malpas JS, Lister TA. The role of transtracheal aspiration in the diagnosis of respiratory infection in neutropenic patients with acute leukaemia. Leuk Res 1981;5:165-8.

${ }^{27}$ Palmer DL, Davidson M, Lusk R. Needle aspiration of the lung in complex pneumonias. Chest 1980;78:1621.

${ }^{28}$ Kalinske RW, Parker RH, Brandt D, Hoeprick PD. Diagnostic usefulness and safety of transtracheal aspiration. $N$ Engl J Med 1967;276:604-8.

${ }^{29}$ Puksa S, Hutcheon MA, Hyland RH. Usefulness of transbronchial biopsy in immunosuppressed patients with pulmonary infiltrates. Thorax 1983;38:146-50.

${ }^{30}$ Webster J, Clarke J. A doctor's dilemma. Chest 1980; 78:417-8. 